\title{
HIPPARCOS, Three years after launch
}

\author{
J. Kovalevsky and M. Froeschlé \\ CERGA - Observatoire de la Côte d'Azur \\ Avenue Copernic \\ F - 06130 Grasse, France
}

\begin{abstract}
In a first part, the present status of the HIPPARCOS mission is described. Despite the degradations and failures of gyroscopes, it is still hoped that a $41 / 2$ mission duration will be reached. The first-year of data has been reduced by both FAST and NDAC consortia. For the best 46200 observed stars, the distribution of standard errors in positions has a maximum of 1.5 mas in latitude and 1.8 mas in longitude and the mean standard error for parallaxes is of the order of 3 mas. The comparison of results obtained by both consortia shows that the differences are small and quite consistent with the announced internal precisions. Magnitude measurements are precise to 0.02 magnitude for a 4 second observation. The precision to be expected for double star observations is also given. The main new result is that the magnitudes of the components are obtained with a few hundredths of a magnitude precision. This allows to devise a new method of mass determination based upon the parallax and a recalibrated mass-luminosity diagram. The parallax dependence of the results is much more favourable than in the case of the classical determination of masses using orbital motions.
\end{abstract}

\section{Payload and satellite status}

The ESA astrometric mission HIPPARCOS was launched on August 8, 1989. Although the launch itself was successful, the failure of the apogee boost motor prevented the satellite to be put on the nominal geosynchronous orbit. Many software modifications were necessary to adapt the mission to the new highly eccentric orbit and two additional telemetry control stations had to be set up. Despite these difficulties, and even before all of them were solved, the continuous operations started on November 27, the effectiveness of the mission being however significantly reduced. Until May 1990, before the Goldstone receiving station became available, useful observations were collected only 40 to $50 \%$ of the time. After June 1990 and until July 1992, the proportion oscillated between 60 and $65 \%$ without any further possibility of being improved. On the other hand, fears concerning the limitation of the life-time due to the degradation of solar panels were found to be unfounded and it 
is expected now that the limit below which the satellite will no more be able to work should not be reached before 1995. At this date, the gas used to control the attitude will be entirely consumed and the present guess is that the mission might continue till mid-94. In any case, ESA has provided financial support for operations until the technical death of the satellite or of its payload.

This of course leaves place for accidents or hardware failures. The most severe experienced until now is the degradation and failure of gyroscopes. There are five gyroscopes. Two, along the $Z$ axis, control the rotation and three are displayed in the $X-Y$ plane. Unfortunately one of the $Z$ gyros failed last year and the second had a major failure in August 1992 that led ESA to put the satellite in a Sun pointing mode unfit for collecting useful scientific data. Two gyro operations have been successfully tested and the collection of data may resume beginning of November. Studies are under way to set up software and procedures for a zero gyro operation in case of a failure of the remaining gyroscopes. But undoubtedly, the efficiency of the observations will be lower than during the last two years. However, if the mission can still remain alive until mid-1994, the precisions for the astrometric parameters may still be of the order of 1.5 mas for parallaxes and 0.9 to 1.2 mas per year for the components of the proper motions.

\section{Principle of HIPPARCOS}

The principle of the payload has been described in many occasions (see for instance, Kovalevsky, 1984; Bernacca, 1985 or Schrijver, 1986). A complete technical description is given in Perryman et al. (1989). Let us only remind that the combined image of two star fields separated by $58^{\circ}$ along the scanning circle is analyzed by a periodic grid while the satellite rotates. An image-dissector successively isolates each star image so that the modulation curves are separated. A star-mapper composed of slits parallel and inclined with respect to the main grid permits to evaluate the direction pointed at in each field of view. The combination of the data acquired during one satellite orbit (a maximum of 9 hours of data) is reduced and one obtains the abscissae of all observed stars in projection on a fixed reference great circle. This is the basic astrometric intermediary result from which all astrometric parameters are to be obtained. Abscissae so obtained are merged in a global solution in which one expresses that the position of a star at a given time is a function of its position at epoch, its proper motion and its parallax. These are the astrometric parameters that are determined in solving simultaneously all the equations of condition for a given star and equations of closure that set all the reference great circles onto a consistent spherical reference system. A general description of the reduction procedures is given in Kovalevsky et al. (1992) for FAST Consortium and in Lindegren et al. (1992) for NDAC.

\section{Results on great circles}

The data acquired by the grid is given in terms of grid coordinates determined from the photon counts in 2.13 second intervals. The precision is of the order of 4 to 5 mas 
for $6^{\text {th }}$ magnitude stars and 14 to 18 mas for stars of magnitude 9 to 10 . In the mean, 30 such coordinates of a given star are used for the solution on a great circle. The resulting precision for abscissae, all magnitudes taken together, is 4 or 3 mas depending on the manner that the attitude is modelled: individual values every 2.13 seconds or smoothed spline functions of time (Van der Marel and Petersen, 1992). The latter method will be the only one left after iterations will be performed.

\section{One year solution for astrometric parameters}

Both NDAC and FAST consortia have treated the first year of data. This is not sufficient to determine good proper motions as, for many stars, the sky coverage is not sufficient to get enough uncorrelated equations. Excluding also double and multiple stars that need additional treatment before astrometric parameters can be computed, we have obtained for FAST positions and parallaxes for about 46700 stars. For these particularly well observed stars, the distribution of standard errors in position is shown in figure 1. It has a maximum around 1.5 mas in latitude and 1.8 mas in longitude (which is not so well determined because of the peculiarities of the scanning law). The mean standard error for parallaxes, whose distribution is given in figure 2, is of the order of 3 mas. Other solutions have also been obtained using different conditions on the choice of stars and of the unknowns as described by Walter et al. (1993).
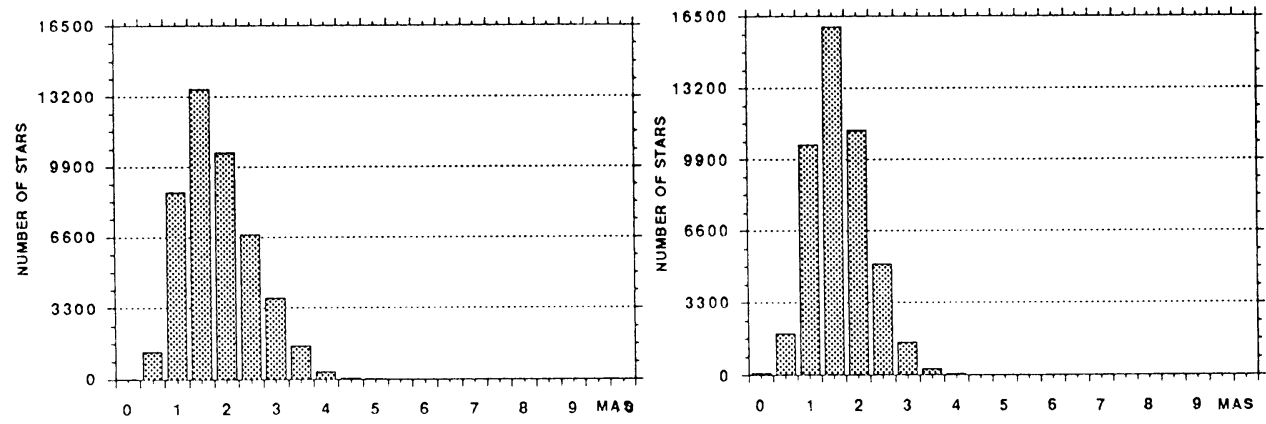

Figure 1 - Distribution of standard errors in ecliptic longitude (left) and ecliptic latitude (right) in FAST one year solution

Approximately the same data was also processed by NDAC. Although the general principles of the reduction are similar, there are significant differences in the procedures and algorithms used. In addition, a preliminary improvement of star positions through star-mapper observations is used by NDAC for attitude reconstitution, so that in a limited sense, NDAC solution is already a kind of second approximation, this being not the case for FAST. 


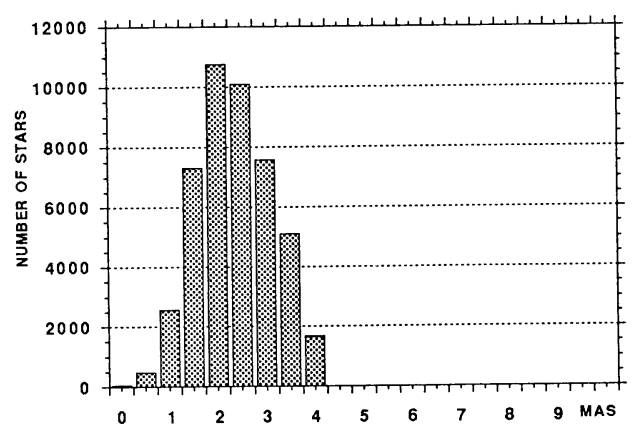

Figure 2 - Distribution of standard errors in parallax in FAST one year solution

\section{Comparison FAST/NDAC}

Both solutions have been compared. Because of the rank deficiency in the sphere reconstitution computed using different algorithms, the resulting reference frames are not the same. They will be redefined and linked to the extragalactic VLBI celestial reference frame only at the end of the reduction. But meanwhile, for comparison sake, it is necessary to apply a rotation to one of the catalogues and reduce it to a common epoch using INCA proper motions and realize a fit by minimizing position differences at the mean epoch of observations.

The comparison was made on 40867 stars common to both solutions with three astrometric unknowns (position and parallax). The rotation found is of the order of 25 mas and was determined with a 0.05 mas precision. The distribution of residuals is by construction centered. The r.m.s. is 1.9 mas in latitude and 2.2 mas in longitude as shown in figure 3.
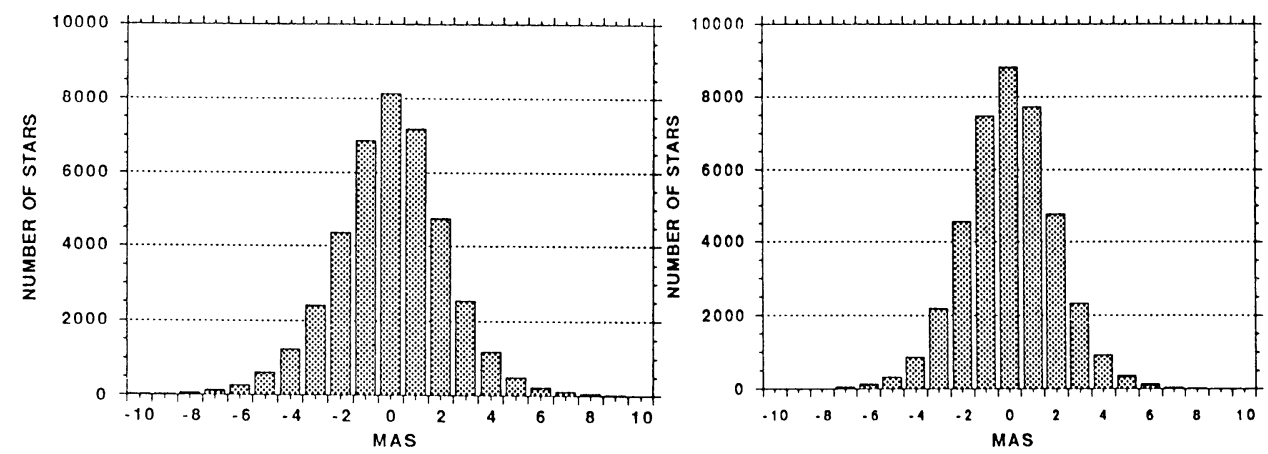

Figure 3 - Distribution of differences NDAC-FAST for 40867 comparison stars in ecliptic longitude (left) and ecliptic latitude (right) in one year solution 
This is fully consistent with the internal precision given by both reductions. Similarly, there is no mean shift in the mean parallax difference whose r.m.s. is 2.5 mas (see figure 4). This is also consistent with the announced precisions. A small systematic North-South effect of the order of 0.1 to 0.2 mas exists. It was actually much larger for comparisons made with less stars in a half-year solution. We believe that they are due to an unsufficient link between the hemispheres because of an unsufficient reference circle coverage, a defect of the scanning law that also shows in the density of stars sufficiently observed to be kept in the solutions (Froeschlé, 1992).

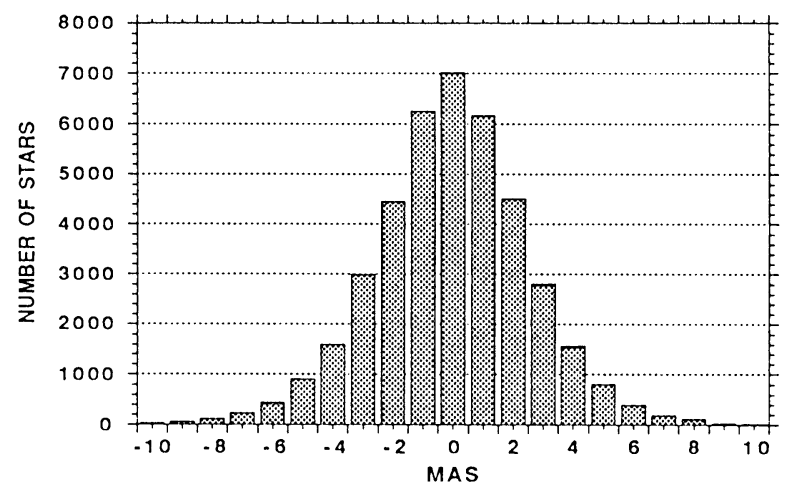

Figure 4 - Distribution of differences in parallax NDAC-FAST for 40867 comparisons stars in one year solution

An examination of the distribution over the sky shows no systematic regional difference between the two solutions except some effects appearing in underobserved regions. We may conclude that no significant difference show up, so that we may have a good confidence on the correctness of both reduction procedures and softwares. They also confirm the expectations already widely publicized on the final precisions to be expected from the mission. Namely, in order to achieve the formal errors predicted for the nominal $2^{1 / 2}$ year mission in parallaxes, one should get data over $4^{1 / 2}$ years while, if the mission stops now, it will be somewhat above 2 mas. In contrast, proper motions that would be of the order of 2 mas per year in longitude and 1.7 mas per year in latitude if the mission stops now, would improve down to 1.1 and 0.9 mas respectively after $4^{1 / 2}$ years of successful observations.

\section{Photometry}

HIPPARCOS is a remarkable wide-band photometer. It is calibrated using magnitudes of more the 11000 stars having an accurate multiband photometry (Grenon et al., 1992). It provides magnitude measurements precise to 0.02 magnitude in one transit, representing about 4 seconds of actual photon counts. The accuracy with respect to the standards is of the same order of magnitude and a remarkable consistency is observed over time. This permits to determine the apparent magnitude of constant 
stars to a few milli-magnitudes and in some cases even a fraction of a millimagnitude. Out of 42000 objects that were the most frequently observed during the first year of operation, $85 \%$ show a precision better than 4 milli-magnitudes. An example is given in figure 5 .

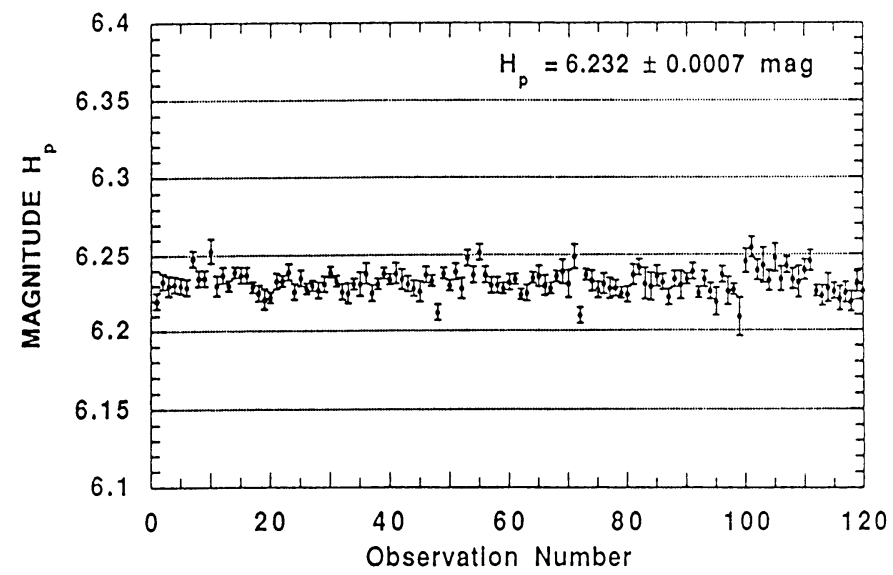

Figure 5 - Example of magnitude determinations of a non variable star. On abscissae are observation numbers that span irregularly one year

In addition, many known or unknown variable stars have been observed. In many cases, the interpretation is difficult because of unfavourable and irregular time observations. But for those variable stars that have continuous stretches of observations during several consecutive days, significant light curves have been obtained (Mignard et al., 1992a).

\section{Double and multiple stars}

The modulated signal of a single star produced by the main grid is symmetrical. But the addition of two such signals with a phase difference as produced by a double star is no more symmetrical and from the evaluation of the phases and intensities of the first two harmonics, one gets informations on magnitudes and projected separation of the components. Combining observations made on different directions, one gets the separation, angle of position and magnitudes of the components of a double star. The precision is of the order of 0 ". 01 in position, and better than 0.02 magnitude for the magnitude of the primary. The precision of the magnitude of the secondary depends greatly on the difference of luminosity of the components as shown in figure 6 (Mignard et al., 1992b). 


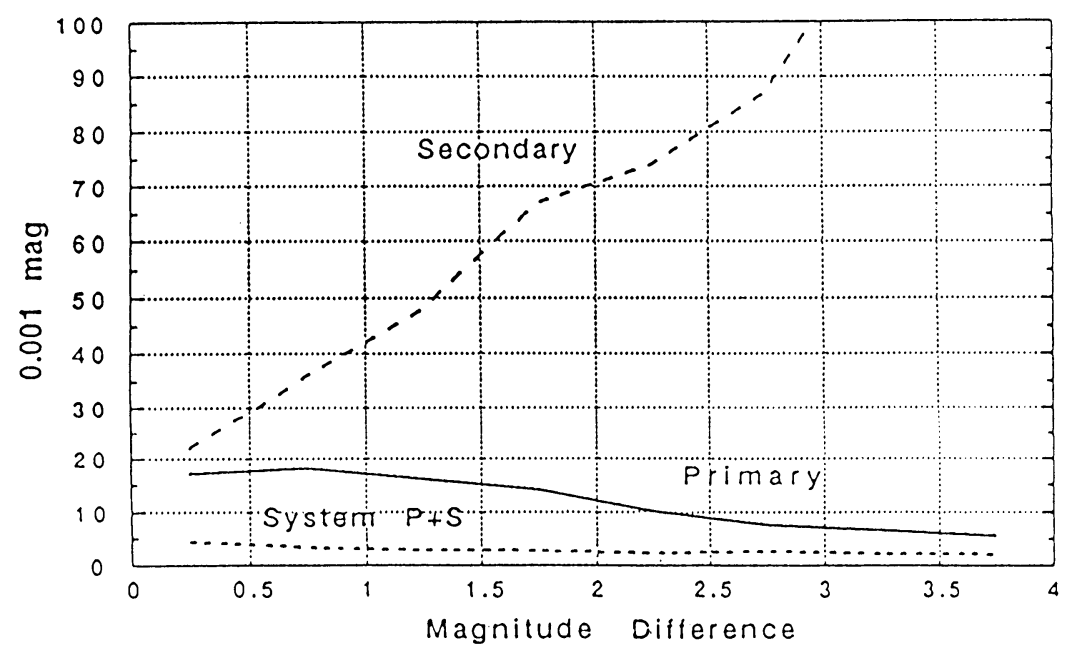

Figure 6 - Precision of magnitude determinations of the components of double stars in function of the difference of their luminosities

External comparisons of the results for some couples with speckle interferometry show no apparent systematic differences and observations lie nicely in the track of earlier observations. An example of the position of HIPPARCOS determination with respect to speckle observations provided by McAllister is given in figure 7 .

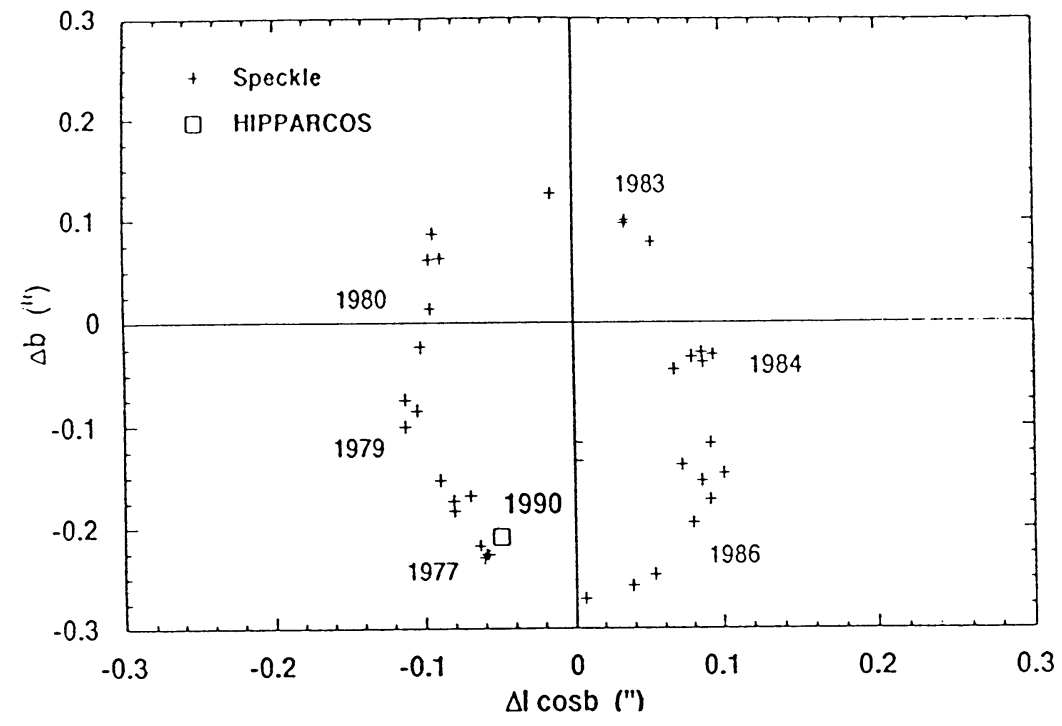

Figure 7 - The HIPPARCOS relative position of the components of a double star, marked by a square lies nicely among speckle observations 
Most of the 11000 stars listed in the Input Catalogue have also been recognized as such by HIPPARCOS and resolved. In addition, several thousands of other double stars have been found. Some of them are present in other catalogues of double stars as Worley's or Couteau catalogues, but many are new. Most have separations of $0 " .1$ to $0 " .3$, a category that is far from being completely recognized from ground based astrometry.

\section{Stellar mass determinations}

The classical approach to the determination of masses of separated double stars is to determine an orbit and deduce the period $\mathrm{P}$ and the apparent semi-major axis a". In order to obtain the sum of masses $\left(\mu_{1}+\mu_{2}\right)$, one must transform a" into astronomical units from the knowledge of the parallax. But the relative error on $\mu_{1}+\mu_{2}$ is three times the relative error on the parallax assuming $\mathrm{a}$ " and $\mathrm{P}$ determined with a negligible contribution to the total error budget. So a $2 \%$ error will be obtained only for stars closer than 4.5 parsecs and $5 \%$ on those whose distance is smaller than 11 parsecs. This leaves a very small number of really useful astrometric masses, if we concur with the statement that for improved astrophysics, one should get a precision of 1 to 2 percents (Andersen, 1991).

In order to get individual masses, one should also have the ratio $\mu_{1} / \mu_{2}$ that is usually obtained from spectroscopic observations. Unfortunately, HIPPARCOS does not help. Not only $\mu_{1} / \mu_{2}$ is not directly accessible from astrometric observations, but one must know it in order to obtain the proper motion of a double star system for which HIPPARCOS gives individual motions of each component. Only in the case when a significant part of the orbit is observed by HIPPARCOS, it is possible to get information on $\mu_{1} / \mu_{2}$, the orbital non linear motion being in this case separated from the global proper motion (Kovalevsky, 1988).

The best method for determining stellar masses is provided by the photometric and spectroscopic observations of eclipsing variables. There are about 45 binary systems that have been throughly observed and gave masses of the components to better than 2\% (Andersen, 1991).

The analysis of these masses and the determination of the absolute luminosity from the distances derived using the double star orbit, showed that there exists two mass-luminosity relations that appear in the mass-luminosity diagram at least for stars more massive than the Sun.

At present, an estimation of these relations is :

$$
\begin{aligned}
& \mathrm{M}_{\mathrm{bol}}=4.6-10 \log \mu \quad \mathrm{M}_{\mathrm{bol}}<7.5 \\
& \mathrm{M}_{\mathrm{bol}}=5.2-6.9 \log \mathrm{M} \quad \mathrm{M}_{\mathrm{bol}}>7.5
\end{aligned}
$$

where $\mathrm{M}_{\mathrm{bol}}$ is the absolute bolometric magnitude and $\mu$ the mass. HIPPARCOS can improve this diagram in using the newly determined parallaxes and extend it for masses smaller than 1 solar mass. The parallax error, appears here without the coefficient 3, while apparent magnitudes have errors of the order of 0.02 magnitudes or less, that is, in luminosity about $2 \%$ or less. HIPPARCOS can therefore recalibrate the mass-luminosity relation in particular in HIPPARCOS magnitudes and discuss 
whether there are only 2 relations, or more, depending on some other astrophysical parameters such as chemical composition or age. The second step is, once this relation is calibrated, to determine new masses of stars. The error budget on parallax $\varpi$ and $\mu$ has respectively, for each branch, the following values

$$
\begin{aligned}
\frac{\varepsilon(\mu)}{\mu} & =0.5 \frac{\varepsilon(\Phi)}{\Phi}+\frac{\varepsilon\left(\mathrm{M}_{\mathrm{bol}}\right)}{23} & & \mathrm{M}_{\mathrm{bol}}<7.5 \\
\frac{\varepsilon(\mu)}{\mu} & =0.7 \frac{\varepsilon(\Phi)}{\Phi}+\frac{\varepsilon\left(\mathrm{M}_{\mathrm{bol}}\right)}{16} & & \mathrm{M}_{\mathrm{bol}}>7.5
\end{aligned}
$$

The error in $\mathrm{M}_{\mathrm{bol}}$ is divided by at least 16 and is negligible. The error in $\mathbb{0}$ is multiplied by 0.7 extending the distance for a given precision in $\mu$ by a factor of 4 in comparison with the astrometric mass determination.

There remains the fact that the HIPPARCOS magnitude is not a bolometric magnitude. One will have to see how accurately one may transform one into another or whether it is better to use a mass-luminosity diagram in terms of HIPPARCOS magnitudes. In addition, spectroscopic determinations of $\mu_{1} / \mu_{2}$ for some double stars will be used to improve the calibration of the mass-luminosity relation.

In conclusion, for the determinations of masses by HIPPARCOS, the most promising procedure comes from the fact that, for the first time, a very accurate magnitude determination of each component is available.

\section{References}

Andersen, J. (1991) "Accurate masses and radii of normal stars", The Astron. and Astrophys., Review 3, 91-126.

Bernacca, P.L. (1985) "Project HIPPARCOS", Astrophys. Space Sci., 110, 21-45.

Froeschlé, M. (1992) CERGA/FAST Technical note $n^{\circ} 54$.

Grenon, M., Mermillod, M. and Mermillod, J.C., 1992, "The HIPPARCOS Input Catalogue, III Photometry", Astron. and Astrophys., 258, 88-93.

Kovalevsky, J. (1984) "Prospects for space stellar astrometry", Space Sci. Rev., 393 163.

Kovalevsky, J. (1988) "Double and multiple star reduction procedures in FAST", in J. Torra and C. Turon (eds.), HIPPARCOS Scientific aspects of the Input Catalogue preparation, II, Published by CIRIT and the Dept. Fisica de l'Atmosfera, Astronomia i Astrofisica, Univ. de Barcelona, 245-255.

Kovalevsky, J., Falin, J.L., Pieplu, J.L., Bernacca, P.L., Donati, F., Froeschlé, M., Galligani, I., Mignard, F., Morando, B., Perryman, M.A.C., Schrijver, H., van Daalen, D.T., van der Marel, H., Villenave, M., Walter, H.G. and 26 other authors (1992) "The FAST HIPPARCOS Data Reduction Consortium: overview of the reduction software", Astron. and Astrophys., 258, 7-17.

Lindegren, L., Hoeg, E., van Leeuwen, F., Murray, C.A. and 12 other authors (1992) "The NDAC HIPPARCOS Data Reduction Consortium: overview of the reduction methods", Astron. and Astrophys., 258, 18-30.

Mignard, F., Froeschlé, M. and Falin, J.L. (1992a) "HIPPARCOS photometry: FAST main mission reduction", Astron. and Astrophys., 258, 142-148. 
Mignard, F., Froeschlé, M., Badiali, M., Cardini, D., Emanuele, A., Falin, J.L. and Kovalevsky, J. (1992b) "HIPPARCOS double star recognition and processing within the FAST Consortium", Astron. and Astrophys., 258, 165-172.

Perryman, M.A.C., Hassan, H. and 22 other authors (1989) "The HIPPARCOS Mission, prelaunch status", The Hipparcos Satellite, ESA Publication, ESA SP-1111, vol. 1.

Schrijver, H. (1986) "HIPPARCOS, the instrument", Manuscripta Geodetica, 11, 97102.

van der Marel, H. and Petersen, C. (1992) "HIPPARCOS great circle reduction, theory, results and intercomparisons", Astron. and Astrophys., 258, 60-69. 